\title{
Experiments on single event electrospraying
}

\author{
Urszula Stachewicz ${ }^{a)}$ and J. Frits Dijksman \\ Philips Research Europe, Eindhoven 5656 AE, The Netherlands \\ Caner U. Yurteri and Jan C. M. Marijnissen \\ DelftChemTech, Delft University of Technology, Delft 2628 BL, The Netherlands
}

(Received 10 October 2007; accepted 29 November 2007; published online 19 December 2007)

\begin{abstract}
In order to use electrospraying as an on demand deposition method, well defined and reproducible single electrospraying events have been studied. To generate a single electrospraying event, rectangular voltage pulses superimposed on a constant bias voltage were applied, and optimum settings of the bias dc voltage for starting such an event were found. This onset point is related to a critical value of the effective surface tension before dripping starts. (c) 2007 American Institute of
\end{abstract} Physics. [DOI: 10.1063/1.2826279]

Over the last twenty years, ink-jet printing technology has been developed steadily to generate droplets ranging from nanoliters to picoliters, the size being restricted to the inner diameter of the nozzles used. Electrohydrodynamic atomization (EHDA), also called electrospraying, is a method suitable for generation of jets and/or droplets with a diameter which is much smaller than the inner diameter of the nozzle. In this letter, we present an approach of EHDA, enabling the generation of droplets and jets with diameter of a few micrometers from a capillary with an outside diameter of $600 \mu \mathrm{m}$ and an inner diameter of $50 \mu \mathrm{m}$.

We want to use EHDA as an on demand volume deposition technology. EHDA refers to a process where a jet breaks up into droplets under the influence of electric forces. ${ }^{1}$ Different spraying modes are possible, but to produce monosized (micron and submicron) droplets, the socalled cone-jet mode is the relevant one, and a constant liquid flow rate is required. ${ }^{2}$ Different authors have reported on the use of EHDA for the controlled deposition of small amounts of liquids. To control the deposited amount, Moerman et $\mathrm{al}^{3}{ }^{3}$ stopped the electrospraying by increasing the distance between the capillary and the substrate. However, they used a continuous flow rate, which cannot be used for electrospraying on demand. Instead, a constant pressure is imposed at the nozzle. Such a system was presented by Yogi et al. ${ }^{4,5}$ who generated a continuous stream of femtoliter droplets, using the cone-jet mechanism. Recent work of Chen et $a l^{6,7}$ showed the possibility of jetting in a similar way. The breakup was avoided by having a short distance between the nozzle and the counterelectrode plate, and the liquid was collected during a single electrical pulse. The total volume emitted $(50-100 \mathrm{pl})$ was proportional to the pulse time. It is not clear what happens when shorter pulses are applied. Another approach was demonstrated by Paine $e t$ al. ${ }^{8}$ In a pulsed electrospray mode, they controlled the number of short duration jet ejections by adjusting the value of the applied voltage pulse. The method gives the possibility of depositing femtoliter volumes of fluid, but it is very sensitive to the applied voltage, because a few extra volts gave a continuous spraying for the same pulse time. This can make the process difficult to control.

${ }^{a)}$ Electronic mail: urszula.stachewicz@philips.com.
In order to have a controlled process for depositing small amounts of fluid, we have to consider the fundamentals of the EHDA mechanism. In particular, we are interested in what happens during the voltage pulse to understand how the amount and the timing of a deposition can be controlled. We want to generate a stable and short event of jetting or droplet formation for each applied voltage pulse of 1-2 ms. We call a fluid deposition during the pulse a single event electrospraying (SEE). To investigate the SEE and to explore the possibilities and limitations of the electrospray technology on demand, an experimental setup was designed and built (Fig. 1). To study the electrospraying process in detail and to achieve well controlled and reproducible single events, rectangular voltage pulses, with pulse time of a few milliseconds, were superposed at low frequencies $(1-10 \mathrm{~Hz})$ on a constant bias voltage. The bias voltage setting is particularly important because of its influence on the liquid meniscus shape in the static situation prior to SEE. For the experiments, ethanol was used because of its low surface tension. The distance between capillary and counterelectrode was $\sim 1 \mathrm{~mm}$. To understand how SEE is generated, all pressures involved when the meniscus is hanging stationary from the capillary in the absence of an electric field were analyzed. For the case the capillary is filled with liquid, we look at the equilibrium of pressures outside and inside the nozzle front.

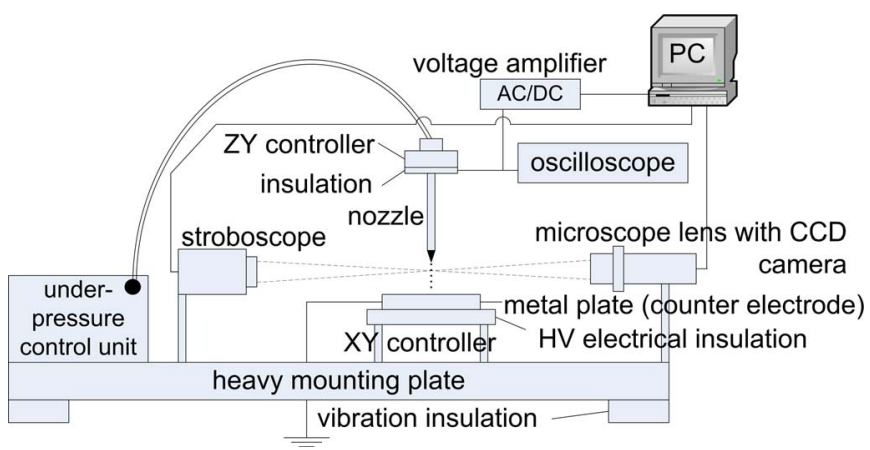

FIG. 1. (Color online) Schematic diagram of the experimental setup. A voltage amplifier is connected to the fluid in a glass capillary by a metal wire inside it. An under-pressure control unit is used to control the pressure. An optical system, consisting of a microscope lens with a progressive scan charge coupled device camera and a stroboscope with adjustable flash delay (illumination system), is used for recording the experiments. The imaging system is triggered by the leading edge of the pulse. The whole setup is mounted on a vibration isolated table. 


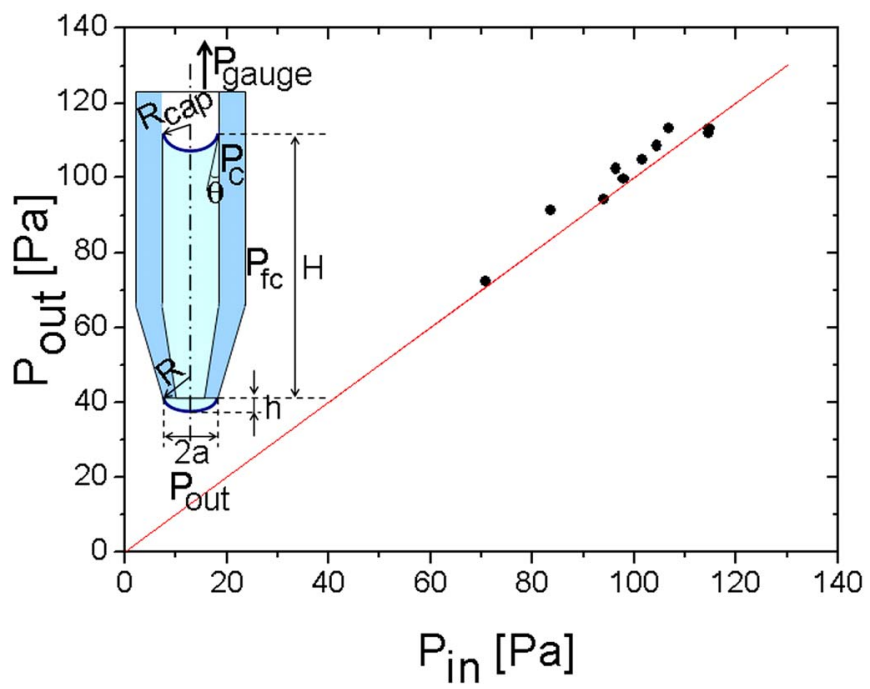

FIG. 2. (Color online) Correlation between the pressure at the end of the capillary $P_{\text {out }}$ and the pressure inside the capillary $P_{\text {in }}$.

Inside, there is the static pressure of the fluid column $P_{\mathrm{fc}}=\rho g H$, where $H$ is the fluid column height, $\rho$ is the density of the liquid, and $g$ is the acceleration due to gravity. In order to keep the system in balance, an under pressure in relation to atmospheric pressure, $P_{\text {gauge }}$ is applied (Fig. 1). With the Young-Laplace relation, the capillary pressure of the meniscus inside the capillary can be calculated, $P_{\mathrm{mc}}$ $=2 \sigma / R_{\text {cap }}$, where $\sigma$ is the surface tension and $R_{\text {cap }}$ the meniscus radius inside the capillary curvature, which depends on the contact angle between the ethanol and the inside of the glass capillary. The contact angle was measured based on pictures of the capillary taken with a microscope. The pressure inside, at the nozzle front is $P_{\mathrm{in}}=P_{\mathrm{fc}}-P_{\mathrm{mc}}-P_{\text {gauge }}$. The pressure caused by the meniscus of the fluid hanging outside the capillary at the same place is $P_{\text {out }}=2 \sigma / R$, where $R$ is the meniscus radius of curvature, which was calculated based on the meniscus height $h$, measured from the pictures taken with our camera system and the outside radius of the nozzle $a$. For static equilibrium at the nozzle, there has to be a pressure balance between $P_{\text {in }}$ and $P_{\text {out }}$ (Fig. 2). The next step is to study the influence of the bias (dc) voltage setting. When the voltage is applied, the meniscus moves downward to a next equilibrium shape (Fig. 3). This change in shape is caused by electrical forces acting on the meniscus, namely, Coulomb repulsion of charge carriers on the meniscus, which can be expressed in terms of reduction of the effective surface tension and attraction of the charged meniscus by the counterelectrode. Surface tension wants to keep the meniscus small and the Coulomb repulsion of the charge carriers on the meniscus wants to increase the meniscus size. The effect of charge on the forces keeping a spherical droplet together in terms of an effective surface tension $\sigma_{\text {eff }}$ has been analyzed by Rayleigh, ${ }^{9}$

$$
\sigma_{\text {eff }}=\sigma-\frac{Q^{2}}{64 \pi^{2} \varepsilon_{0} \varepsilon_{r} R^{3}},
$$

where $Q$ is the total charge on the droplet surface, $\varepsilon_{r}$ is the relative permittivity of the air, and $\varepsilon_{0}$ is the vacuum permittivity. We assume that formula (1) is also valid for the meniscus as long as the surface charge density stays the same. The charge on the meniscus $Q_{\text {men }}$ can be estimated by the equation $Q_{\text {men }}=C U$, where $U$ is the applied voltage and $C$ is Downloaded 10 Aug 2010 to 131.180.130.114. Redistribution subje

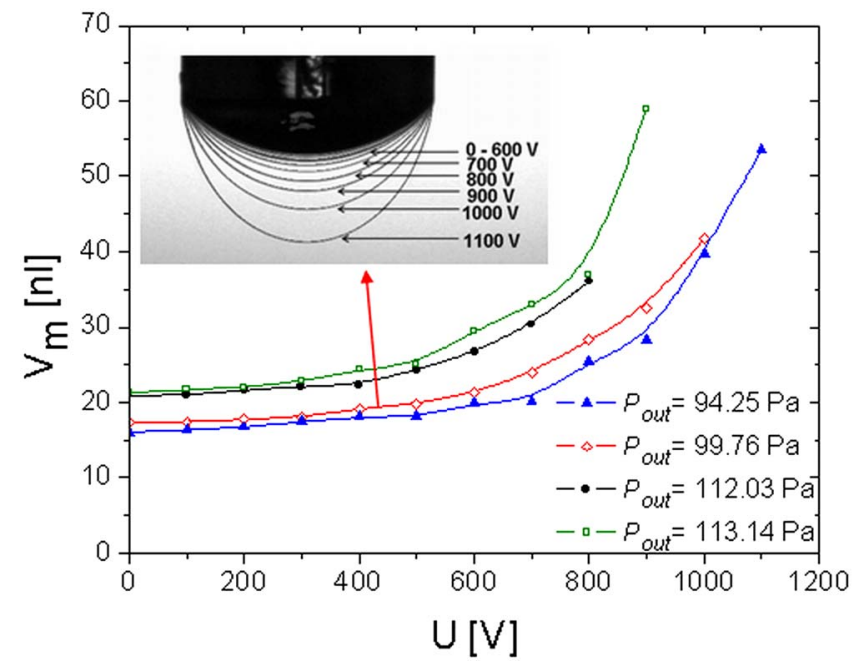

FIG. 3. (Color online) Correlation between the applied bias voltage $U$ and the volume $V_{m}$ of the meniscus in equilibrium (upper limit, just before dripping starts). Results were obtained for four different meniscus pressures $P_{\text {out }}$.

the capacity of the system. Further, we assume that the meniscus is a part of a flat plate capacitor, so that $C=\varepsilon_{0} \varepsilon_{r} A / d$, with $A=2 \pi R h$ is the surface area of the meniscus and $d$ is the distance between the liquid meniscus and the counterelectrode. As a result, $Q_{\text {men }}=Q h / 2 R$, thus $Q=2 R C U / h$. Substitution in Rayleigh's formula (1) yields the second term in Eq. (2). In terms of pressure, corresponding to effective surface tension, we have $P_{\mathrm{ST}}=2 \sigma_{\text {eff }} / R$. In addition, there is the pressure related to the attractive Coulomb forces between the meniscus and the counterelectrode, again considering the system as a flat plate capacitor and the meniscus as a flat plate, $P_{\mathrm{CF}}=U^{2} C / 2 d \pi a^{2}$. We see that $P_{\mathrm{CF}}$ decreases the effective surface tension further. Consequently, by adding $P_{\mathrm{ST}}$ and $P_{\mathrm{CF}}$, we can define a total effective surface tension as

$$
\sigma_{\text {eff-total }}=\sigma-\left(\frac{C^{2}}{16 \pi^{2} \varepsilon_{0} \varepsilon_{r} R h^{2}}+\frac{C R}{4 \pi d a^{2}}\right) U^{2} .
$$

We have assumed that the other forces such as gravity can be neglected. This explains the quadratic behavior of the total effective surface tension $\sigma_{\text {eff-total }}$ as a function of applied voltage $U$. Figure 4 indicates a very good fit between the theoretical and experimental values of $\sigma_{\text {eff-total }}$. As can be seen, there is a deviation between experimental and theoretical results for potentials higher than $700 \mathrm{~V}$. This is caused by the fact that for the theoretical curve, a constant value of the distance between meniscus and counterelectrode is used. The applied voltage was gradually raised. Every potential increment leads to volume increase and reshaping of the meniscus. When a critical potential is reached, the meniscus is in transition to the dripping mode. This is the bias voltage used as a control point for starting the SEE. Using the same bias voltage, pulses with different width and voltage values were applied to achieve the single events. For a meniscus pressure of $112 \mathrm{~Pa}, 1000 \mathrm{~V}$ was the bias voltage to start applying the pulses. Then the pulse height and duration were optimized accordingly. At a pulse height in the range of around 400-600 V, a steady cone jet appeared and a highly reproducible SEE was reached in this setting range. Therefore, by increasing the flash delay in between consecutive pictures and taking one picture per event, we were able to see all steps of the whole process. During a voltage pulse, the to AIP license or copyright; see http://apl.aip.org/apl/copyright.jsp 


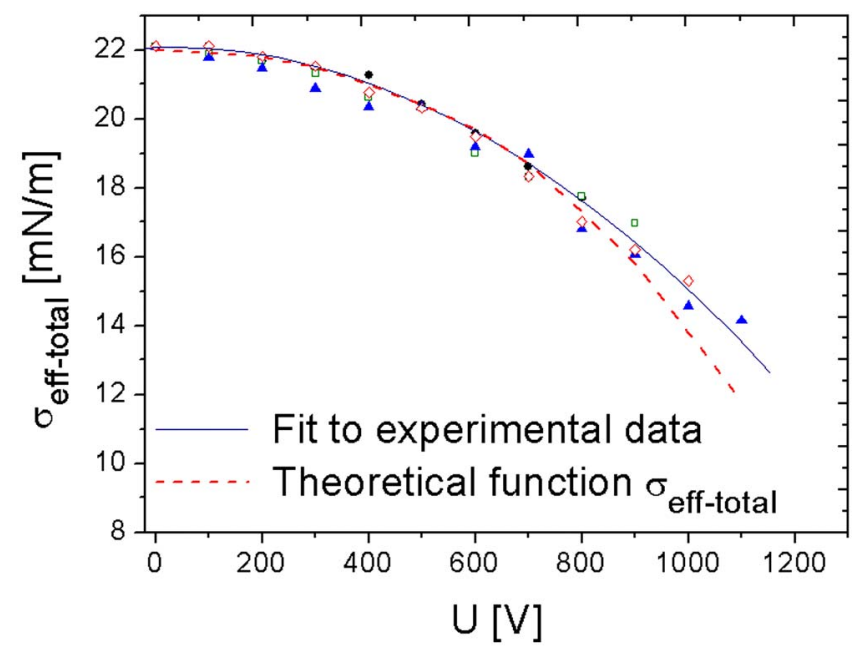

FIG. 4. (Color online) Experimental and theoretical total effective surface tensions $\sigma_{\text {eff-total }}$ of ethanol as a function of applied voltage $U$. Results were obtained for four different meniscus pressures, as in Fig. 3.

equilibrium meniscus of the liquid changes rapidly into a cone, and subsequently into a jet formed at the cone apex (Fig. 5). Then, in some cases the jet breaks up into fine droplets (not seen in Fig. 5) and after a while, the spraying/ jetting stops and the meniscus returns to its equilibrium shape. Based on images taken during SEE, we were able to affirm that the generated droplet and jet sizes were in the range of 5-18 $\mu \mathrm{m}$ in diameter. We are able to monitor all different steps during SEE. This makes it possible to improve the quality of the process and obtain the optimum settings for a future technology development. In comparison to previous work, ${ }^{5,7,8}$ this study uses a larger meniscus. Consequently, a bias voltage to create reproducible SEE is needed. Additionally, we are able to apply shorter pulses. With our way of monitoring, we see many details such as cone development, jet breakup, and droplet size.

In SEE, the electric field is used to control the meniscus shape. We notice that pictures taken with the same flash delay from different events are almost identical. This means that the SEE was reproduced for each applied pulse. By selecting a suitable bias voltage and pulse, we provide a

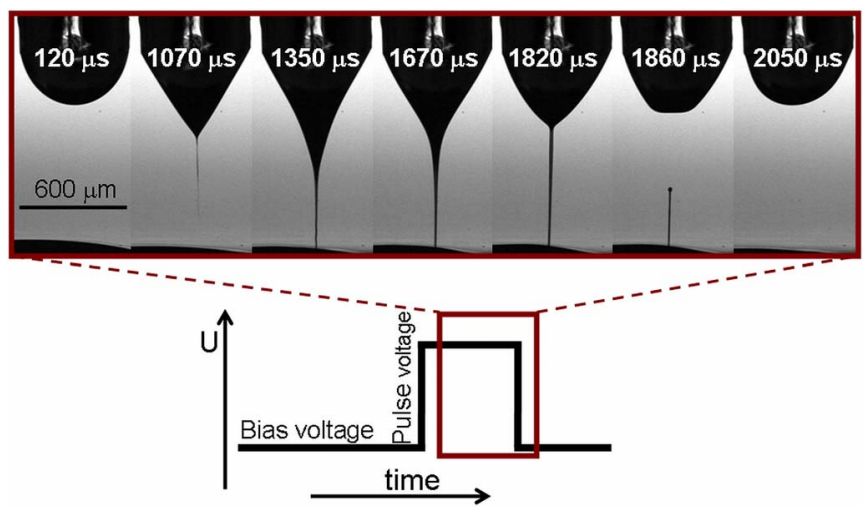

FIG. 5. (Color online) Jet formation during single event electrospraying of ethanol. The measurements are taken at a bias voltage of $1 \mathrm{kV}$ with a $2 \mathrm{~ms}$ block pulse of $0.6 \mathrm{kV}$ every $300 \mathrm{~ms}$, with the glass capillary placed at a distance of around $1 \mathrm{~mm}$ from the counterelectrode. The deposited liquid on the pictures is from few single events.

method of generating well controlled SEE. Further work includes the reduction of volume of fluid ejected per single event electrospraying.

This study is supported by the European research program, Marie Curie Actions, Early Stage Fellowship under Project No. MEST-CT-2004-505006 and Philips Research Europe. The authors thank Peter Barendse, Albert Geven, Martin Vernhout, and Leo van den Besselaar for the assistance and help with the building the setup.

${ }^{1}$ R. P. A. Hartman, D. J. Brunner, D. M. A. Camelot, J. C. M. Marijnissen, and B. Scarlett, J. Aerosol Sci. 30, 823 (1999).

${ }^{2}$ J. M. Grace and J. C. M. Marijnissen, J. Aerosol Sci. 25, 1005 (1994).

${ }^{3}$ R. Moerman, J. Frank, J. C. M. Marijnissen, T. G. Schalkhammer, and G. W. van Dedem, Anal. Chem. 73, 2183 (2001).

${ }^{4}$ O. Yogi, T. Kawakami, and A. Mizuno, Anal. Chem. 76, 2991 (2001).

${ }^{5}$ O. Yogi, T. Kawakami, and A. Mizuno, J. Electrost. 64, 634 (2006).

${ }^{6}$ C. H. Chen, D. A. Saville, and I. A. Aksay, Appl. Phys. Lett. 88, 154104 (2006).

${ }^{7}$ C. H. Chen, D. A. Saville, and I. A. Aksay, Appl. Phys. Lett. 89, 124103 (2006).

${ }^{8}$ M. D. Paine, M. S. Alexander, K. L. Smith, M. Wang, and J. P. Stark, J. Aerosol Sci. 38, 315 (2007).

${ }^{9}$ J. W. S. Rayleigh, The Theory of Sound (Macmilllan and Co., New York, 1896), Vol. II, p. 364. 